\title{
Classification of M2-brane 2-torus bundles, $U$-duality invariance, and type II gauged supergravities
}

\author{
Maria Pilar Garcia del Moral* \\ Departamento de Física, Universidad de Antofagasta, Aptdo 02800, Chile \\ J. M. Pena ${ }^{\dagger}$ \\ Departamento de Física, Facultad de Ciencias, Universidad Central de Venezuela, \\ Apartado Postal 47270, Caracas 1041-A, Venezuela \\ Alvaro Restuccia \\ Departamento de Física, Universidad de Antofagasta, Aptdo 02800, Chile
}

(Received 31 December 2018; published 10 July 2019)

\begin{abstract}
We obtain the complete classification of the inequivalent classes of M2-brane symplectic torus bundles with monodromy in $S L(2, Z)$ and the precise $U$-duality relations among them. There are eight inequivalent classes of bundles whose monodromy groups, at low energies, are in correspondence with the gauging groups of the eight type II gauged supergravities in nine dimensions. Four of those have previously been found, and they correspond to the "type IIB side." We provide the explicit realization of the remaining four classes associated with the "type IIA side." The precise M2-brane $U$-duality relations between the eight inequivalent classes of bundles have allowed us to identify the remaining four. We conjecture that the classes of gaugings-classifying the eight types of II gauged supergravity in nine dimensions - are determined by the inequivalent coinvariant classes associated with the base and the fiber of the supermembrane bundles and their duals.
\end{abstract}

DOI: 10.1103/PhysRevD.100.026005

\section{INTRODUCTION}

M-theory is a theory candidate for unification of all the interactions in nature that contains supermembrane theory-also called M2-brane theory-as one of its building blocks. Any quantum consistent definition of M-theory will require its understanding. Supermembranes are $(2+1)$-dimensional objects embedded in 11-dimensional (11D) space-time that act as sources for $D=11$ supergravity, and as a consequence, there is a deep relation between both theories: on the one hand, supermembrane theory is conjectured to contain a unique massless groundstate associated with the 11D supergravity multiplet. Several works have been developed in support of this claim; for a recent new approach see Ref. [1] and references

*maria.garciadelmoral@uantof.cl

jpena@fisica.ciens.ucv.ve

Present address: Departamento de Fisica, Universidad de Antofagasta, Aptdo 02800, Chile.

alvaro.restuccia@uantof.cl

Published by the American Physical Society under the terms of the Creative Commons Attribution 4.0 International license. Further distribution of this work must maintain attribution to the author(s) and the published article's title, journal citation, and DOI. Funded by SCOAP . therein. On the other hand, supermembrane theories are expected to be described by supergravities at "low energies." In that respect, the M-theory origin of maximal supergravities in any dimension $d \leq 11[2,3]$ is well known to correspond to the 11D supermembrane compactified on a trivial $T^{11-d}$-torus [4].

There are other types of supergravity theories, like gauged/massive supergravities. Gauged supergravities can be obtained from string/supergravity theories in a number of ways: by compactifying on manifolds with nontrivial holonomy [5,6], by flux compactifications [7], by gauging procedures like the embedding tensor $[8,9]$, by ScherkSchwarz (SS) reductions [10] where the reduced fields keep a nontrivial phase dependence on the internal spatial coordinates [11-13], or as effective descriptions of string/M-theory formulated on nontrivial torus bundles [14-16]. In the torus bundle formulation the internal dependence of SS fields becomes associated with a twist given by a monodromy $\mathcal{M}_{\text {sugra }}$ of the bundles [17-19].

In contrast, the determination of the M-theory action origin of the gauged/massive supergravity deformations has become much more elusive. The M-theory uplift of SS nine-dimensional reduction was conjectured to be related to torus bundles with monodromy in $S L(2, Z)$ [17]. The action must be an invariant functional formulated in terms 
of the local sections of this bundle. In Ref. [20] Garcia del Moral et al. showed that the supermembrane formulated on 2-torus bundles with monodromy in $S L(2, Z)$ is the origin of type II gauged supergravities in nine dimensions. Type II supergravities in nine dimensions consist of a unique maximal supergravity and eight gauged deformations [11-13], four of them coming from the type IIA sector, and the remaining four from the type IIB one. At effective level, their 10D origins are the type IIB supergravity, the maximal type IIA, and the two type IIA massive deformations: Romans [21] and Howe et al. [22]. In this paper we extend the analysis of the M2-brane $T_{U}$-duality transformation done in Ref. [20], where the four inequivalent M2-brane 2-torus bundles associated with the "type IIB" sector were found an the other four were inferred from the $T_{U}$-duality invariance of the mass operator. We provide an explicit construction of the four inequivalent classes of M2-brane bundles associated with the "type IIA" side in nine dimensions.

Another topic that has received a lot of attention from the community are the $T$-dual $/ U$-dual invariant theories in string and M-theory in order to have a better insight of the nonperturbative structure of these theories. $U$ duality is a nonperturbative transformation defined in 11D that uplifts and unifies the perturbative $T$ duality and nonperturbative $S$ duality present in 10D. For the case of M-theory compactified on a 2-torus, the $U$-duality group is conjectured to be $S L(2, Z) \times Z_{2}$. M-theory as a unification theory should contain them as symmetries, and its effective description should be invariant. In the context of effective field theories, they have been constructed in terms of modified supergravity actions enriched with terms of stringy origin in which $T$ duality is manifest. These attempts have focused mainly on two different approaches: double field theory [23-28] and generalized geometry [29-31] focusing either on its stringy action $[30,32]$ or on the M-theory realization [33-35]. It has even been possible to incorporate the $\alpha^{\prime}$ corrections into the analysis in recent times [36] as well as into winding contributions from the very beginning [37]. See Refs. [38-41] for a review.

Global aspects of the $T$ duality are relevant and should also be considered in order to achieve $U$-dual invariant actions at the level of M-theory. Some of them were studied in Refs. [32,42]. In this paper we want to exhaustively characterize the M2-branes on a 2-torus bundle (with and without monodromy) which is the simplest nontrivial example of $U$-dual invariant theories-at the level of mass operators-connected with type II maximal and gauged supergravities. We focus on the characterization of the M2-brane bundle description, extending the results found in Ref. [20]. The supermembrane bundle class is specified through the coinvariants of the fiber and the base. We emphasize the role played by the coinvariant of the base manifold in the bundle structure when the $U$-duality action is performed. We will denote $S_{U}$ duality and $T_{U}$ duality in quotation marks in the text when we want to stress the particular action of 11D $U$ duality that connects with its counterpart ( $S, T$ duality) when reduced to $10 \mathrm{D}$. As we will see in Sec. II, $S$ duality is a symmetry of the mass operator of the supermembrane; hence to verify the $U$-duality properties we will focus on the $T_{U^{\text {-duality }}}$ action. $T_{U^{-}}$ duality maps M2-brane torus bundles onto M2-brane torus bundles, preserving the invariance of the Hamiltonian and mass operator but interchanging the cohomological charges of the base manifold with the homological ones of the fiber at the same time that the monodromy is mapped onto a dual monodromy that belongs to the same conjugation class. We analyze the case where the $U$-duality group corresponds to $S L(2, Z) \times Z_{2}$, and the $U$-dual invariant orbit of charges is classified by the coinvariant class of the M2-brane bundle. This orbit is completely filled with the charges associated with M2-branes. Consequently under $U$ duality the M2brane 2-torus bundles are mapped onto M2-brane 2-torus bundles, and $U$ duality does not include charges associated with branes of different dimensionality, in contrast to the case in which more compactified dimensions are considered $[43,44] . U$ duality interchanges those invariants; however, the duality map among the coinvariants does not correspond to an equivalence relation. $U$ duality does not act on generic grounds linearly. The $U$-duality action on the global structure of the bundle is the relevant one to explain the difference observed at effective level between the two sectors of type II gauged supergravity in 9D from an M-theory point of view. On the supergravity side the gauging groups of the type IIA and type IIB do not coincide, so $U$ duality does not preserve them either $[11,12]$. This fact is totally natural at the level of effective field theory since the global $S L(2, R)$ symmetry is not realized on the type IIA side at perturbative level. We discuss their possible relation with the M2-brane torus bundles in Sec. IV, and in the discussion section.

The paper is structured as follows: In Sec. II we review the formulation of the supermembrane theory on torus bundles with monodromy in $S L(2, Z)$, and we show that the mass operator is $S_{U}$-dual invariant. In Sec. II A we extend the analysis previously done for the M2-brane 2-torus bundle by studying the role played by the coinvariant $C_{B}$ associated with the base manifold. In Sec. III we describe in detail the $T_{U}$-duality transformation for the supermembrane bundle, understanding by it the $U$-duality part associated with $T$ duality in 10D when the M2-brane theory is double dimensionally reduced. We specify its local and global action on the 2-torus bundles according to its coinvariant classification. In Sec. IV we obtain explicitly, by analyzing the action of $T_{U}$ duality on the coinvariant structures, the four inequivalent classes of bundles of M2-brane bundles. We conjecture that they are associated at low energies with the gaugings in the type IIA supergravity sector in nine dimensions. In Sec. V we present a discussion of the role of the $U$-duality part associated when 
double dimensionally reduced to $T$ duality to explain the differences in the bundle structure and in the deformations allowed at supergravity level from the supermembrane point of view, and finally we present our conclusions. In Appendix A we describe some of the technical properties of the coinvariants of bundles with monodromy, and in Appendix B we deduce the most general supermembrane $T_{U}$-duality transformation.

\section{SUPERMEMBRANE THEORY ON A SYMPLECTIC TORUS BUNDLE}

In this section we will review the supermembrane theory compactified on an $M_{9} \times T^{2}$ formulated globally on symplectic torus bundles. These bundles are classified according to two inequivalent topological sectors: they can be principal (i.e., with trivial monodromy) or nontrivial with a monodromy group contained in $S L(2, Z)$. The supermembrane theory in the light cone gauge (LCG) has a residual gauge symmetry, the symplectomorphisms on the base manifold which in two dimensions are equivalent to the area preserving diffeomorphisms (APDs). When the theory is formulated globally, the group of symplectomorphisms corresponds to the structure group of the torus bundle. It is well known that supermembrane theory on a torus when double dimensionally reduced corresponds to the type IIB superstring compactified on a circle $[45,46]$. The supermembrane compactified on a trivial torus is associated at low energies with a type II maximal supergravity in nine dimensions [45]. When the compactification is nontrivial but associated with a central charge condition, the theory is described at low energies by the type IIB gauged supergravities as shown in Ref. [20].

Let us review the construction of the symplectic M2-brane torus bundle in this first part of the section. The Hamiltonian of a supermembrane theory with central charges formulated in the LCG on a target space $M_{9} \times T^{2}$, the supermembrane subject to a central charge condition, is the following one [47-53]:

$$
\begin{aligned}
H= & \int_{\Sigma} T_{\mathrm{M} 2}^{2 / 3} \sqrt{\rho}\left[\frac{1}{2}\left(\frac{P_{n}}{\sqrt{\rho}}\right)^{2}+\frac{1}{2} \frac{P \bar{P}}{\sqrt{\rho}}+\frac{T_{\mathrm{M} 2}^{2}}{4}\left\{X^{m}, X^{n}\right\}^{2}\right]+\int_{\Sigma} T_{\mathrm{M} 2}^{2 / 3} \sqrt{\rho}\left[\frac{T_{\mathrm{M} 2}^{2}}{2}\left(\mathcal{D} X^{n}\right)\left(\overline{\mathcal{D}} X^{n}\right)\right]+\int_{\Sigma} T_{\mathrm{M} 2}^{2 / 3} \sqrt{\rho}\left[+\frac{T_{\mathrm{M} 2}^{2}}{4}(\mathcal{F} \overline{\mathcal{F}})\right] \\
& +\left(\mathrm{n}^{2} \mathrm{Area}_{T^{2}}^{2}\right)+\int_{\Sigma} T_{\mathrm{M} 2}^{2 / 3} \sqrt{\rho}\left[-\bar{\Psi} \Gamma_{-} \Gamma_{n}\left\{X^{n}, \Psi\right\}-\frac{1}{2} \bar{\Psi} \Gamma_{-} \bar{\Gamma} \mathcal{D} \Psi-\frac{1}{2} \bar{\Psi} \Gamma_{-} \Gamma \overline{\mathcal{D}} \Psi\right] \\
& +\int_{\Sigma} \sqrt{\rho} L\left[\frac{1}{2} \overline{\mathcal{D}}\left(\frac{P}{\sqrt{\rho}}\right)+\frac{1}{2} \mathcal{D}\left(\frac{\bar{P}}{\sqrt{\rho}}\right)+\left\{X^{n}, \frac{P_{n}}{\sqrt{\rho}}\right\}-\left\{\bar{\Psi} \Gamma_{-}, \Psi\right\}\right]
\end{aligned}
$$

where $L$ is a Lagrange multiplier and $T_{\mathrm{M} 2}$ is the 11D tension of the supermembrane, and $\rho$ is the determinant of a nonflat 2-torus $\Sigma$ that corresponds to the spatial part of the world-volume metric. The symplectic bracket is defined as $\{\mathrm{A}, \mathrm{B}\}=\omega^{a b} \partial_{a} \mathrm{~A} \partial_{b} \mathrm{~B}$, whose symplectic 2-form is $\omega=$ $\omega^{a b} d \sigma_{a} \otimes d \sigma_{b}$ with $\omega^{a b}=\frac{i \varepsilon^{a b}}{2 \sqrt{\rho}}$, with $a, b=z$, $\bar{z}$ being the local complex coordinates and its complex conjugate $\bar{z}$, respectively, defined on the base manifold $\Sigma . X^{n}$ are the embedding maps $\Sigma \rightarrow M_{9}$, where $n=3, \ldots, 9$ and $X=$ $X^{1}+i X^{2}$ are the embedding ones from $\Sigma \rightarrow T^{2}$. They are scalars parametrizing the transverse coordinates of the supermembrane in the target space. $P_{n}$ are densities, and they are the canonical momenta associated with the $X^{n}$ and $P$, respectively, of the field $X . \Psi$ are scalars on the world volume but an $S O(7)$ spinor on the target space, $\Gamma_{n}$ are seven gamma matrices, and $\Gamma=\Gamma_{1}+i \Gamma_{2}$, denoting by $\bar{\Gamma}$ its complex conjugate. The 2-torus $T^{2}$ of the target space is characterized by the moduli $\mathrm{R}$ as the radius, and $\tau$ as the complex Teichmüller parameter. The winding numbers are $l_{s}, m_{s}$, with $r, s=1,2$ associated with the wrapping of the supermembrane on the $T^{2}$. They define a matrix $\mathbb{W}=\left(\begin{array}{ll}l_{1} & l_{2} \\ m_{1} & m_{2}\end{array}\right)$. When the wrapping is irreducible, its determinant $\mathrm{n}$ is other from zero [51], and the theory has discrete spectrum [52], in contrast to the wrapped supermembrane without this topological condition. This sector defines a topological condition associated with the existence of world-volume monopoles that algebraically imply the existence of a nonvanishing central charge in the supersymmetric algebra. For this reason this sector was called the supermembrane with central charges. On this sector there is a symplectic curvature defined on the base manifold as $\mathcal{F}=D \bar{A}-\bar{D} A+\{A, \bar{A}\}$, with $A$ being a connection under the infinitesimal symplectomorphism transformation $\delta_{\varepsilon} A=\mathcal{D} \varepsilon$. See Refs. [47,54] for a detailed analysis. The symplectic covariant derivative is defined as $\mathcal{D} \bullet=D \bullet+\{A, \bullet\}$, with $D \bullet=e_{r}^{a} \partial_{a} \bullet$ being a rotated covariant derivative $[20,55]$ defined in terms of a zweibein $e_{r}^{a}$ as

$$
e_{r}^{a}:=-2 \pi \mathrm{R}\left(l_{r}+m_{r} \tau\right) \Theta_{s r} \omega^{b a} \partial_{b} \widehat{X^{s}},
$$

with $r, s=1,2 . d \hat{X}$ being the harmonic 1-form basis defined on $\Sigma$. The Hamiltonian is invariant, and the residual symmetry under APDs connected and not connected to the identity.

The Hamiltonian is subject to the APD group residual constraints (connected to the identity $\phi_{1}$, but also to the large APD $\left.\phi_{2}\right)$ 
$\phi_{1}: d\left(\frac{1}{2}(P d \bar{X}+\bar{P} d X)+P_{m} d X^{m}-\bar{\Psi} \Gamma_{-} \Psi\right)=0$,

$\phi_{2}: \oint_{\mathcal{C}_{r}}\left[\frac{1}{2}(P d \bar{X}+\bar{P} d X)+P_{m} d X^{m}-\bar{\Psi} \Gamma_{-} d \Psi\right]=0$

where $\mathcal{C}_{s}$ is the canonical 1-homology basis on $T^{2}$.

The theory is invariant under two different $\operatorname{SL}(2, Z)$ discrete symmetries: the first one is associated with the invariance under the change of the basis of the harmonic 1 -forms defined on the Riemann world volume [46] and the windings

$$
d \hat{X} \rightarrow S d \hat{X}, \quad \mathbb{W} \rightarrow S^{-1} \mathbb{W},
$$

with $S \in S L(2, Z)$. This dependence is encoded in the matrix $\Theta \in S L(2, Z)$ [55] in Eq. (2).

The second one is associated with an invariance of the mass operator involving $S L(2, Z)$ symmetry related to the target 2-torus $T^{2}$, so it is invariant under $S$-duality transformations

$$
\begin{aligned}
\tau & \rightarrow \frac{a \tau+b}{c \tau+d}, \\
\mathrm{R} & \rightarrow \mathrm{R}|c \tau+d|, \\
A & \rightarrow A e^{i \varphi_{\tau}}, \\
\mathbb{W} & \rightarrow\left(\begin{array}{cc}
a & -b \\
-c & d
\end{array}\right) \mathbb{W}, \\
\mathbf{Q} & \rightarrow\left(\begin{array}{ll}
a & b \\
c & d
\end{array}\right) \mathbf{Q},
\end{aligned}
$$

where $\left(\begin{array}{l}a b \\ c d\end{array}\right) \in S L(2, Z)$ and $c \tau+d=|c \tau+d| e^{-i \varphi_{\tau}}$, and where $\mathbf{Q}=\left(\begin{array}{l}p \\ q\end{array}\right)$ is the Kaluza-Klein (KK) charge of the supermembrane propagating on the target 2-torus considered. The homological charges of the target torus $H_{1}\left(T^{2}\right)$ are interpreted as the quantized $\mathrm{KK}$ charges of the compactified supermembrane [20].

Now we formulate the previous embedding description in terms of a symplectic torus bundle with monodromy in $S L(2, Z)$. This global formulation is going to make manifest some topological invariants that carry physical information. The total space $E$ is defined in terms of a fiber $F=M_{9} \times T^{2}$ and $\Sigma$ as the base manifold. The structure group $\mathrm{G}$ is the symplectomorphism leaving invariant the canonical symplectic structure in $T^{2}$. The action of G on $F$ produces a $\pi_{0}(\mathrm{G})$-action on the homology and cohomology of $F$. The monodromy $\mathcal{M}_{G}$ is defined as

$$
\mathcal{M}_{G}: \pi_{1}(\Sigma) \rightarrow \pi_{0}(\mathrm{G})
$$

with

$$
\mathrm{G}=\operatorname{Symp}\left(T^{2}\right) \quad \text { and } \quad \pi_{0}(\mathrm{G})=\operatorname{SL}(2, Z) .
$$

Consequently $\mathcal{M}_{G}=\left(\begin{array}{ll}a b \\ c d\end{array}\right)^{\gamma} \in S L(2, Z)$, and it acts on the homology basis of the $T^{2}$ target torus-where $\gamma=\gamma_{1}+\gamma_{2}$ with $\left(\gamma_{1}, \gamma_{2}\right)$ are the integers characterizing the element of the homotopic group $\pi_{1}(\Sigma)$. The symplectic connection defined on the base manifold transforms with the monodromy $d A \rightarrow d A e^{i \varphi_{\mathcal{M}_{G}}}$, where $\varphi_{\mathcal{M}_{G}}$ is a discrete monodromy phase given by $\varphi_{\mathcal{M}_{G}}=\frac{c \tau+d}{|c \tau+d|}$ for a given modulus $\tau$. The inequivalent classes of symplectic torus bundles over $\Sigma$ are classified by the elements of the second cohomology group, $H^{2}\left(\Sigma, Z_{\mathcal{M}_{G}}^{2}\right)$ or equivalently by their coinvariants. See $[20,54,56]$ for more details. The global symmetries of the theory become restricted by the monodromy.

\section{A. The role of fiber $C_{F}$ and base $C_{B}$ coinvariants on the bundle structure}

The supermembrane symplectic torus bundles are characterized by two types of coinvariants relevant to the characterization of the supermembrane bundle. The class of coinvariants associated with the fiber $C_{F}$ and the coinvariants associated with the base $C_{B}$. The torus bundles with a given monodromy $\mathcal{M}_{G}$ are classified according to the elements of the twisted second cohomology group $H^{2}\left(\Sigma, Z_{\mathcal{M}}^{2}\right)$ of the base manifold $\Sigma$. Its coefficients are defined on the module generated by the monodromy representation acting on the homology of the target torus [56]. There is a bijective relation with the elements of the coinvariant group $C_{F}=\left\{C_{\mathbf{a}}\right\}, a=1, \ldots, j$ associated with a particular monodromy group $\mathcal{M}_{G}$; see Appendix A for more properties of the coinvariant classes. A coinvariant class in the KK sector is given by

$$
C_{F}=\left\{\mathbf{Q}+\left(\mathcal{M}_{g}-\mathbb{I}\right) \hat{\mathbf{Q}}\right\}
$$

for any $g \in \mathcal{M}_{G}$, and $\hat{\mathbf{Q}}$ is any arbitrary element of the KK sector. That is, two elements belong to the same class if they differ in an element $(g-1) \hat{\mathbf{Q}}$ for some $g \in \mathcal{M}_{G}$ and $\hat{\mathbf{Q}}$.

Associated with the monodromy subgroup $\mathcal{M}_{G}$ there is an induced action on the cohomology of the base manifold [57], which corresponds to the monodromy group of the winding sector $\mathcal{M}_{G}^{*}$. Since $\mathcal{M}_{G}^{*}=\Omega \mathcal{M}_{G} \Omega^{-1}$ with $\Omega=\left(\begin{array}{cc}-1 & 0 \\ 0 & 1\end{array}\right)$, it lies in the same conjugacy class of $\mathcal{M}_{G}$. $\mathcal{M}_{G}^{*}$ acts on the fields which define the Hamiltonian, that is, on sections of the torus bundle through a matrix $\Theta=$ $\left(V^{-1} \mathcal{M}_{G}^{*} V\right)^{T}$ that appears in the symplectic covariant derivatives $\mathcal{D}_{r}$ of the Hamiltonian $H . V$ is associated with the monodromy subgroup $\mathcal{M}_{G} \subset S L(2, Z)$; see Ref. [20] for further details. Induced by them there are two other possible invariants $\left(\mathcal{M}_{G}^{*}, C_{B}\right)$ characterizing the symplectic bundles. The two monodromies lie in the same conjugation 
class; however, they are not the same, and consequently their respective coinvariants are not equivalent.

A coinvariant class in the winding sector is given by

$$
C_{B}=\left\{\mathbf{W}+\left(\mathcal{M}_{g}^{*}-\mathbb{I}\right) \hat{\mathbf{W}}\right\},
$$

with $\mathcal{M}_{g}^{*} \in \mathcal{M}_{G}^{*}$ being the monodromy group acting in the winding sector specified by $\mathbf{W}=\left(\begin{array}{l}l \\ m\end{array}\right) \in H^{1}(\Sigma)$. Because of the $S L(2, Z)$ symmetry on the equivalence class of the basis of homology on the base manifold (a torus), it is possible to reduce the problem to work indistinctly with the winding matrix $\mathbb{W}$, or with $\mathbf{W}$ defined by the first row of the matrix W. See Appendix A. Then for M2-brane or symplectic torus bundles one needs to specify both types of coinvariants for a given monodromy $\mathcal{M}_{G}$, those associated with the fiber $C_{F}$ and those associated with the base manifold $C_{B}$. Given a symplectic torus bundle, the Hamiltonian of the theory and the mass operator are defined on the coinvariant classes.

The dependence of the bundle on the winding charges $\mathbf{W}$ and the $\mathrm{KK}$ charges $\mathbf{Q}$ is defined in terms of the function $\mathrm{F}$ :

$$
\mathrm{F}: \mathbf{W} \rightarrow \mathbf{W}, \quad \mathbf{Q} \rightarrow \mathbf{Q},
$$

which depends on the classes $C_{B}$ and $C_{F}$. Since these are invariant under the action of the monodromy, the same occur for the Hamiltonian or the mass operator. We thus have

$$
\mathrm{F}\left(C_{B}\right)=\mathrm{F}(\mathbf{W}), \quad \mathrm{F}\left(C_{F}\right)=\mathrm{F}(\mathbf{Q}) .
$$

In particular if we consider the action only on the orbits instead of the coinvariant classes, $\mathrm{F}$ may be defined in terms of a matrix $\Theta$ acting on the matrix $\mathbb{W}: \Theta \mathbb{W}$ in a way that, under the monodromy, $\mathbb{W} \rightarrow \mathcal{M}^{*} \mathbb{W}$ and $\Theta \rightarrow \Theta\left(\mathcal{M}^{*}\right)^{-1}$, as we have defined the coinvariant derivative. A way to obtain a function $F$ with such a property is to consider a linear function $F_{L}$ defined on the orbits of the elements of the coinvariant class:

$$
\mathrm{F}_{L}\left(C_{B}\right)=\mathrm{F}_{L}\left(\mathbf{W}+\left(\mathcal{M}^{*}-\mathbb{I}\right) \hat{\mathbf{W}}\right)=\mathrm{F}_{L}(\mathbf{W}) .
$$

Each coinvariant class is invariant under the action of any $g \in \mathcal{M}_{G}$. So the coinvariant class may be considered itself as a class of orbits under the action of $\mathcal{M}_{G}$. Given a symplectic torus bundle, the Hamiltonian of the theory is defined for any orbit of the coinvariant class.

\section{III. $T_{U}$ DUALITY FOR SUPERMEMBRANE THEORY TORUS BUNDLES}

Disclaimer: in this section we analyze the action of $U$ duality over the M2-brane torus bundle that in 10D corresponds to $T$ duality when the M2-brane is double dimensionally reduced. In other words we focus on the part of the $U$-duality action that exchanges winding charges and Kaluza-Klein charges and acts on the moduli at the same time that it transforms the structure of the torus bundle. In order to emphasize these aspects, we also called it by the name of $T_{U}$ duality even when we are working at the level of $11 \mathrm{D}$.

The $T_{U}$-duality transformation acts on the Hamiltonian $H$ and the mass operator $\mathbf{M}^{2}$, and it also has an action on the topological invariants of the M2-brane torus bundle describing the theory. In what follows, we are going to describe the $T_{U}$-duality action at global level, that is, by specifying its action on the supermembrane symplectic torus bundle structure, and secondly we will describe its action on the mass operator of the supermembranes.

\section{A. $T_{U}$-duality action on the coinvariants}

The $T_{U}$-duality transformation globally transforms a bundle into a dual one by interchanging the cohomological charges of the torus base manifold into the homological charges defined on the torus fiber with dual moduli. $T_{U}$ duality also interchanges the coinvariant class of the base and the fiber in the dual torus bundle:

$$
\left(C_{F}, C_{B}\right)=\left(\tilde{C}_{B}, \tilde{C}_{F}\right)
$$

where $\tilde{C}$ denotes the dual coinvariant class. It may occur, however that the transformation becomes nonlinear. At low energies this fact will be reflected in the change of the gauging group associated with the corresponding dual supergravity. In order to delve into this classification, we are going to characterize the action of $T_{U}$ duality over the different classes of M2-brane bundles with monodromies trivial and nontrivial:

(a) Trivial monodromy: We first consider the case in which $\mathcal{M}_{G}=\mathbb{I}$, i.e., when the monodromy group is trivial. In this case the coinvariant classes, which classify the inequivalent torus bundles, have only one element $\mathbf{Q}$ in the $\mathrm{KK}$ sector, and one element $\mathbf{W}$ in the winding sector. The $T_{U}$-dual transformation is defined in terms of $\mathcal{T} \in S L(2, Z)$ with equal diagonal terms, satisfying

$$
\tilde{Q}=\mathbf{W}=\mathcal{T} \mathbf{Q}, \quad \tilde{W}=\mathbf{Q}=\mathcal{T}^{-1} \mathbf{W},
$$

where $\mathcal{T}=\left(\begin{array}{l}\alpha \beta \\ \gamma \alpha\end{array}\right) \in S L(2, Z)$ is defined as in Appen$\operatorname{dix}$ B. Given $\mathbf{Q}$ and an associated winding matrix $\mathbb{W}$, there always exists a winding matrix on the equivalence class of $\mathbb{W}$ defined by the action from the right by $\mathbb{S} \in S L(2, Z)$ such that

$$
\mathbb{W} \mathbb{S}=\mathcal{T} \mathbb{Q},
$$

where $\mathbb{Q}$ is the matrix whose first row is $\mathbf{Q}$, and where it also has determinant $n$. Given $\mathbf{Q}$, there always exists $\mathbb{Q}$, though it is not unique. The most general one is 
obtained by multiplying from the right by a parabolic matrix with integer coefficients and equal diagonal elements:

$$
\mathbb{Q} \mathbb{K}, \quad \mathbb{K}=\left(\begin{array}{ll}
1 & k \\
0 & 1
\end{array}\right) .
$$

The matrix $\mathbb{K}$ can always be absorbed into $\mathbb{S}$. The symplectic torus bundles are classified in this case, i.e., $\mathcal{M}_{G}=\mathbb{I}$, by two integers, the elements $Z \otimes Z$. The symplectic torus bundles are in one-to-one correspondence to the $U(1) \times U(1)$ principle bundle over the base manifold. Since the monodromy is trivial, the structure group may reduce to the group of symplectomorphism homotopic to the identity. The dual transformation is then completed by the transformation of the moduli given in Eq. (22).

(b) Nontrivial monodromy: We now consider the case where the monodromy group $\mathcal{M}_{G}$ is nontrivial. It is an Abelian subgroup of $S L(2, Z)$. The $T_{U}$-dual transformation maps as before coinvariant classes on the KK sector onto the coinvariant class in the winding sector. In order to define the $T_{U}$-duality map, we take any element $\mathbf{Q}$ of $C_{F}$ and any element $\mathbf{W}$ of $C_{B}$ and map them as in Eq. (15). The global $T_{U}$-duality map is given by the following transformation:

$$
\begin{aligned}
& \mathbf{Q} \stackrel{\mathcal{T}}{\rightarrow} \mathbf{W}, \quad \mathcal{M}_{G} \stackrel{\Omega}{\rightarrow} \Omega \mathcal{M}_{G} \Omega^{-1}, \\
& \hat{\mathbf{Q}} \stackrel{f}{\rightarrow} \hat{\mathbf{W}}, \quad C_{F} \stackrel{\mathbb{T}}{\rightarrow} C_{B},
\end{aligned}
$$

where $f$ is a general linear map from the $\hat{\mathbf{Q}}$ sector onto the $\hat{\mathbf{W}}$ sector. We denote by $\mathbb{T}$ the $T_{U}$-duality action on the coinvariants that can either act linearly $\mathcal{T}$ or not. In particular the map $f$ can be defined as

$$
\hat{\mathbf{W}}=\mathcal{T} \hat{\mathbf{Q}} .
$$

However, in general it is not necessary to relate $f$ to $\mathcal{T}$.

Suppose now that instead of mapping $\mathbf{Q}$ to $\mathbf{W}$, we map it to another member of the coinvariant class to which $\mathbf{W}$ belongs:

$$
\mathbf{Q} \stackrel{\mathcal{T}}{\rightarrow} \mathbf{W}+\left(\mathcal{M}_{G}^{*}-\mathbb{I}\right) \hat{\mathbf{W}}_{1}
$$

then

$$
\begin{aligned}
C_{F} & =\left\{\mathbf{Q}+\left(\mathcal{M}_{G}-\mathbb{I}\right) \hat{\mathbf{Q}}\right\} \stackrel{\mathbb{T}}{\rightarrow}\left\{\mathbf{W}+\left(\mathcal{M}_{G}^{*}-\mathbb{I}\right)\left(\hat{\mathbf{W}}+\hat{\mathbf{W}}_{1}\right)\right\} \\
& =C_{B} .
\end{aligned}
$$

That is, the new map is only a translation on the $\hat{\mathbf{W}}$ sector, and the map $f$ includes a translation by $\hat{\mathbf{W}}_{1}$. Equation (21) shows that changing the map for
$\mathbf{Q} \rightarrow \mathbf{W}$ to $\mathbf{Q} \rightarrow\left(\mathbf{W}+\left(\mathcal{M}_{G}^{*}-\mathbb{I}\right) \hat{\mathbf{W}}\right)$ is equivalent to leaving the map $\mathbf{Q} \rightarrow \mathbf{W}$ and changing $f$ by a translation. The translation by $\hat{\mathbf{W}}_{1}$ is irrelevant from the point of view of the coinvariant class since $\hat{\mathbf{W}}+\hat{\mathbf{W}}_{1}$ is a general element of the winding sector. Hence the map between the coinvariant classes is determined only by $\mathcal{T}$, which is constructed from one element of each class, $\mathbf{Q}$ and $\mathbf{W}$, respectively. The generator $\mathcal{M}_{G}$ can be parabolic, elliptic, or hyperbolic.

\section{B. $T_{U}$-duality action on the mass operator}

The duality transformation on the symplectic torus bundle has an action not only on the charges but also on the geometrical moduli. We define dimensionless variables $\mathcal{Z}$, where $\mathcal{Z}=\left(T_{\mathrm{M} 2} \mathrm{~A} Y\right)^{1 / 3}$ with $\mathrm{A}=(2 \pi \mathrm{R})^{2} \operatorname{Im} \tau$, as the area of the target torus, and $Y=\frac{\mathrm{RIm} \tau}{|q \tau-p|}$ as a variable proportional to the $\mathrm{R}$ radius of the complex torus. $Y$ is invariant under the monodromy group if we consider $\mathbf{Q}$ the components of $\mathbf{F}(\mathbf{Q})$. The $T_{U}$-duality transformation is given by

$$
\begin{array}{cc}
\text { the moduli: } \mathcal{Z} \tilde{\mathcal{Z}}=1, & \tilde{\tau}=\frac{\alpha \tau+\beta}{\gamma \tau+\alpha}, \\
\text { the charges: } \tilde{\mathbf{Q}}=\mathcal{T} \mathbf{Q}, & \tilde{\mathbf{W}}=\mathcal{T}^{-1} \mathbf{W},
\end{array}
$$

with $\alpha, \beta, \gamma$ being the integer entries of the $\mathcal{T}$ matrix in Eq. (15). The charges $\mathbf{Q}, \mathbf{W}$ transform depending on the type of bundle considered, i.e., with trivial or nontrivial monodromy. We notice that $\mathcal{Z}, Y$, and their duals are invariant on an orbit generated by $\mathcal{M}_{G}$ contained in the respective coinvariant classes, provided that $\tau$ and $\mathbf{Q}$ transform as in Eq. (6). Moreover, they are independent of the coinvariant class when we define $Y$ in terms of the components of $\mathbf{F}(\mathbf{Q})$ instead of $\mathbf{Q}$ and leave $\tau$ as a invariant moduli under monodromy. The symmetry of the Hamiltonian related to the basis of harmonic 1-forms of the Riemann world volume [46] allows us to define the class of orbits associated with the winding matrices $[\mathbf{W}]$. Following Eq. (15), there always exists a $\mathcal{T}$ such that

$$
\mathcal{T} \mathbf{Q}=[\mathbf{W}], \quad \mathcal{T}^{-1} \mathbf{W}=[\mathbf{Q}],
$$

such that $T$-duality maps classes onto classes

$$
[\mathbf{W}] \rightarrow[\tilde{\mathbf{Q}}]=[\mathbf{W}], \quad[\mathbf{W}] \rightarrow[\tilde{\mathbf{W}}]=[\mathbf{Q}]
$$

Let us recall $[20,57]$ that the relation between the radius modulus and its dual follows from Eq. (22) and was obtained in

$$
\tilde{\mathrm{R}}=\frac{|\gamma \tau+\alpha||q \tau-p|^{2 / 3}}{T_{\mathrm{M} 2}^{2 / 3}(\operatorname{Im} \tau)^{4 / 3}(2 \pi)^{4 / 3} \mathrm{R}} .
$$


$T_{U}$ duality defines a nonlinear transformation on the charges of the supermembrane since $\mathcal{T}$ is constructed from them, in contrast to the usual $S L(2, Z)$ action on the moduli, which is a linear one. This property will be very relevant for understanding the dual multiplet structure. The KK modes are mapped onto the winding modes, and vice versa, as expected. This property together with the condition $\mathcal{Z} \tilde{Z}=1$ ensures that $(T \text { duality })^{2}=I$. This transformation becomes a symmetry for $\mathcal{Z}=\tilde{\mathcal{Z}}=1$ which imposes a relation between the tension, the moduli, and the KK charges of the wrapped supermembrane,

$$
T_{\mathrm{M} 2}^{0}=\frac{|q \tau-p|}{\mathrm{R}^{3}(\operatorname{Im} \tau)^{2}} .
$$

Given the values of the moduli, it fixes the allowed tension $T_{\mathrm{M} 2}^{0}$ - or the other way around, for a fixed tension $T_{\mathrm{M} 2}^{0}$, the radius, the Teichmüller parameter of the 2-torus, and the KK charges satisfy Eq. (26). For $\mathcal{Z}=1$ the Hamiltonian and the mass operator of the supermembrane with central charges are invariant under $T_{U}$ duality:

$$
\begin{aligned}
\mathbf{M}^{2} & =\left(T_{\mathrm{M} 2}^{0}\right)^{2} \mathrm{n}^{2} \mathrm{~A}^{2}+\frac{k^{2}}{Y^{2}}+\left(T_{\mathrm{M} 2}^{0}\right)^{2 / 3} H \\
& =\frac{\mathrm{n}^{2}}{\tilde{Y}^{2}}+\left(T_{\mathrm{M} 2}^{0}\right)^{2} k^{2} \tilde{\mathrm{A}}^{2}+\left(T_{\mathrm{M} 2}^{0}\right)^{2 / 3} \tilde{H}
\end{aligned}
$$

with $H=\tilde{H}$. See Ref. [20] for further details. In Ref. [57] the authors showed that there always exists a $\mathcal{T}$, a parabolic matrix transformation of $T_{U}$ duality given for any arbitrary value of the KK and winding charges. This parabolic transformation depends on the winding and KK momenta of the supermembrane bundle. In this work we extend this analysis to characterize in detail the most general $T_{U^{-}}$ duality transformation $\mathcal{T}$; see Appendix B. In principle the $T_{U}$-duality transformations are a subset of the parabolic, elliptic, and hyperbolic transformations with equal diagonal terms. We show there that the parabolic one plays a distinguished role since it is the only class able to map any kind of winding and $\mathrm{KK}$ charges for a given supermembrane torus bundle with arbitrary monodromy and general central charge onto its dual. If we restrict the central charge to $\mathrm{n}=1$, then all of the different types of $T_{U}$-dual transformations $\mathcal{T}$ are allowed, i.e., elliptic $\mathcal{T}_{e}$, parabolic $\mathcal{T}_{p}$, and hyperbolic ones $\mathcal{T}_{h}$; however, since the supermembrane can be wrapped any arbitrary number of times a 2 -torus, there is no justification to restrict it to $n=1$. For $n \neq 1$ the situation is different: the $\mathcal{T}_{Z_{4}}$ elliptic case of $T_{U}$-duality transformations fail to map the torus bundles except for very specific KK and $\mathrm{W}$ charges. In contrast, the hyperbolic $T_{U}$-duality matrices $\mathcal{T}_{h}$ always exist for arbitrary $\mathbf{Q}, \mathbf{W}$, and $\mathrm{n}$. The difference with respect to the parabolic case $\mathcal{T}_{p}$ relies on the fact that for each set of charges there is needed a different hyperbolic realization with a different trace. For a general transformation, the parabolic $T_{U}$ duality one, $\mathcal{T}_{p}$, is going to be the one responsible for characterizing the different M2-torus bundles dual which at low energies are associated with the different type II gauged supergravities in nine dimensions; for that reason we will denote $\mathcal{T}_{p} \equiv \mathcal{T}$.

\section{CLASSIFICATION OF $U$-DUAL SUPERMEMBRANE BUNDLES}

In this section we are going to establish the precise correspondence between the type IIA side of the supermembrane bundle with parabolic, elliptic, hyperbolic, and trombone monodromies. We will focus on the $T_{U}$-duality action analyzed in the preceding section. The M2-brane bundle analysis explains this fact since the $T_{U}$-dual transformation in general does not commute with the monodromy group (except for the parabolic monodromy case), and consequently its associated $T_{U}$-dual coinvariant class of the bundle does not lie in the same equivalence class as the original one. This happens even though the monodromy and its dual are in the same conjugation class as was originally signaled in Ref. [58].

The $T_{U}$-dual transformation maps a given charge $\mathbf{Q}$ in the KK sector onto a winding $\mathbf{W}=\mathcal{T} \mathbf{Q}$, and the coinvariant class of $\mathbf{Q}$ onto the coinvariant class of $\mathbf{W}$. That is, the coinvariant class

$$
C_{F} \equiv\left\{\mathbf{Q}+\left(\mathcal{M}_{G}-\mathbb{I}\right) \hat{\mathbf{Q}}\right\}
$$

is mapped onto

$$
\tilde{C}_{F}=\left\{\mathbf{W}+\left(\mathcal{M}_{G}^{*}-\mathbb{I}\right) \hat{\mathbf{W}}\right\}=C_{B}
$$

on the winding sector, which may or may not coincide with

$$
\mathcal{T} C_{F}=\left\{\mathcal{T} \mathbf{Q}+\mathcal{T}\left(\mathcal{M}_{G}-\mathbb{I}\right) \mathcal{T}^{-1}(\mathcal{T} \hat{\mathbf{Q}})\right\}
$$

because generically

$$
\mathcal{M}_{G}^{C}:=\mathcal{T} \mathcal{M}_{G} \mathcal{T}^{-1} \neq \mathcal{M}_{G}^{*}=\Omega \mathcal{M}_{G} \Omega^{-1} .
$$

If both classes define the same coinvariant class, i.e., $\tilde{C}_{F}=\mathcal{T} C_{F}$, then for any element of the matrix group monodromy $\mathcal{M}_{g_{1}}^{*}$ and $\hat{\mathbf{W}}$

$$
\left(\mathcal{M}_{G}^{C}-\mathbb{I}\right) \mathcal{T} \hat{\mathbf{Q}}-\left(\mathcal{M}_{g_{1}}^{*}-\mathbb{I}\right) \hat{W}=\left(\mathcal{M}_{g_{2}}^{*}-\mathbb{I}\right) \hat{\hat{\mathbf{W}}}
$$

for some $\mathcal{M}_{g_{2}}^{*}, \hat{\hat{\mathbf{W}}}$ denotes an arbitrary element in the winding sector. $\mathcal{M}_{g_{1}}^{*}, \mathcal{M}_{g_{2}}^{*} \in \mathcal{M}_{G}^{*}$. In this case, the $T_{U}$ -duality map transforms the symplectic torus bundles with a given monodromy group into themselves. Generically that is not the case, i.e., $\tilde{C}_{F} \neq \mathcal{T} C_{F}$, so we have to study in detail 
what occurs for each type of bundle of monodromy: parabolic, elliptic, hyperbolic, and trombone.

\section{A. $\boldsymbol{U}$-dual of M2-brane parabolic torus bundles}

The M2-brane torus bundles with parabolic monodromies has two inequivalent nontrivial monodromies classes,

$$
\mathcal{M}_{p}=\left(\begin{array}{ll}
1 & \mathrm{p} \\
0 & 1
\end{array}\right), \quad \mathcal{M}_{Z_{2}}=\left(\begin{array}{cc}
-1 & 0 \\
0 & -1
\end{array}\right)
$$

satisfying $\left|\operatorname{Tr}\left(\mathcal{M}_{p}\right)\right|=2$. While associated with the first type of monodromy, the coinvariants are generically torsionless [the only exception is the $(0,0)$ class associated with the torsion one], in the second type of parabolic monodromy all coinvariants have torsion and the KK charges are restricted to $(0,0),(0,1),(1,0),(1,1)$. A coinvariant with torsion determines a symplectic torus bundle whose local symplectic structure on the fibers is the restriction of the global symplectic one. For the parabolic case the dual monodromy group is given by $\mathcal{M}_{p}^{C}=$ $\mathcal{T} \mathcal{M}_{g} \mathcal{T}^{-1}=\mathcal{M}_{p}$ since $\mathcal{T}$ commutes with $\mathcal{M}_{p}$. In addition the group $\mathcal{M}_{p}^{*}=\Omega \mathcal{M}_{p} \Omega^{-1}$ coincides with the group $\mathcal{M}_{p}$ since $\Omega g \Omega^{-1}=g^{-1}$ for any $g \in \mathcal{M}_{p}$. The condition (32) is then satisfied. The $T_{U}$-duality map acts linearly, and it transforms the class of the M2-brane symplectic torus bundles with parabolic monodromy group into itself. The same occurs for $\mathcal{M}_{Z_{2}}$. They are the unique conjugation class of bundles satisfying this property. At low energies we conjecture that they correspond to a parabolic gauged supergravity on the type IIA side. This 9D gauged supergravity is associated with the KK reduction of the 10D Romans supergravity [13].

\section{B. $U$-duals of M2-brane elliptic and hyperbolic torus bundles}

We will analyze the two cases separately, and we will see that under $T_{U}$ duality they generate a unique class of supermembrane dual torus bundles.

The elliptic monodromy group $\mathcal{M}_{e}$ [59] is finitely generated by the following matrices,

$$
\begin{aligned}
\mathcal{M}_{Z_{3}} & =\left(\begin{array}{cc}
0 & 1 \\
-1 & -1
\end{array}\right)^{\gamma}, \\
\mathcal{M}_{Z_{6}} & =\left(\begin{array}{cc}
1 & 1 \\
-1 & 0
\end{array}\right)^{\gamma},
\end{aligned}
$$

satisfying $\left|\operatorname{Tr}\left(\mathcal{M}_{e}\right)\right|<2$ with $\gamma \in Z$. Their associated coinvariant of the fiber equivalence classes can be computed explicitly using Eq. (9). For the $Z_{3}$ case there are three classes

$$
C_{Z_{3}}^{F}=\left\{\mathbf{Q}_{i}+\left(\mathcal{M}_{Z_{3}}-\mathbb{I}\right) \hat{\mathbf{Q}}\right\}, \quad i=1,2,3,
$$

with

$\mathbf{Q}_{1}=\left(\begin{array}{l}0 \\ 0\end{array}\right), \quad \mathbf{Q}_{2}=\left(\begin{array}{l}0 \\ 1\end{array}\right), \quad \mathbf{Q}_{3}=\left(\begin{array}{l}0 \\ 2\end{array}\right)$

It can be shown that Eq. (32) cannot be satisfied. In fact Eq. (32) means that, for any $\hat{g} \in \mathcal{M}_{Z_{3}}^{C}$ and $\hat{\mathbf{Q}}$, there exist $g \in \mathcal{M}_{Z_{3}}^{*}$ and $\hat{\mathbf{W}}$ such that

$$
\left(\widehat{\mathcal{M}}_{g}-\mathbb{I}\right) \mathcal{T} \hat{\mathbf{Q}}=(g-\mathbb{I}) \hat{\mathbf{W}}
$$

One can show that there does not exist $\hat{\mathbf{W}}$ satisfying the equality (37) for suitable $\hat{g} \in \mathcal{M}_{Z_{3}}^{C}$ and $\hat{\mathbf{Q}}$. In the same way it can be shown that Eq. (32) is not satisfied for the monodromy groups $\mathcal{M}_{Z_{4}}, \mathcal{M}_{Z_{4}}^{*}$.

In contrast, Eq. (32) is satisfied for $\mathcal{M}_{Z_{6}}$ and $\mathcal{M}_{Z_{6}}^{*}$. This means that given any element $\hat{g} \in \mathcal{M}_{Z_{6}}^{C}$ and any $\hat{\mathbf{Q}}$, there exists $g \in \mathcal{M}_{Z_{6}}^{*}$ and $\tilde{\mathbf{W}}$ satisfying Eq. (32), and vice versa. This follows because one element of the group is $g_{Z_{6}}=\left(\begin{array}{cc}1 & 1 \\ -1 & 0\end{array}\right)$; hence $\left(g_{Z_{6}}^{*}-\mathbb{I}\right)$ has a determinant equal to 1 , and then there always exists a $\tilde{W}$ satisfying Eq. (32):

$$
\left(g_{Z_{6}}^{*}-\mathbb{I}\right)^{-1}\left(\tilde{g}_{Z_{6}}^{*}-\mathbb{I}\right) \mathcal{T} \hat{\mathbf{Q}}=\tilde{\mathbf{W}} .
$$

The other way round follows in the same way: given any $g \in \mathcal{M}_{Z_{6}}^{*}$ and $\tilde{\mathbf{W}}$, there always exists a $\hat{\mathbf{Q}}$ satisfying Eq. (37). In fact

$$
\operatorname{det}\left(\mathcal{T} g_{Z_{6}} \mathcal{T}^{-1}-\mathbb{I}\right)=1
$$

and we proceed as before. The coinvariant Abelian group associated with the monodromy $\mathcal{M}_{Z_{6}}$ has only one element in the KK sector given by the class

$$
C_{Z_{6}}^{F}=\left\{\mathbf{Q}_{0}+\left(\mathcal{M}_{Z_{6}}-\mathbb{I}\right) \hat{\mathbf{Q}}\right\}
$$

where $\mathbf{Q}_{0}$ is any particular charge and the only one element in the winding sector, respectively.

Let us now analyze the case of hyperbolic monodromy. There are infinite Abelian monodromy groups of hyperbolic matrices constructed in terms of

$$
\mathcal{M}_{h}=\left(\begin{array}{ll}
a & b \\
c & d
\end{array}\right)^{\gamma}
$$

such that $\left|\operatorname{Tr}\left(\mathcal{M}_{h}\right)\right|>2$ with $\mathcal{M}_{h} \in S L(2, Z)$ [59]. It can also be explicitly shown that generically the coinvariant structure of the dual bundle is not equivalent to the original one.

Let us now compare the dual monodromies of elliptic and hyperbolic 2-torus bundles with respect to their 
conjugate classes. We consider the generic monodromy case $g \in \mathcal{M}_{G}$ with $g=\left(\begin{array}{l}a b \\ c d\end{array}\right) \in S L(2, Z)$. Then

$$
\mathcal{T} g \mathcal{T}^{-1}=\left(\begin{array}{cc}
a+t c & -t(a+t c)+b+t d \\
c & -c t+d
\end{array}\right) .
$$

By defining with $u=a-d$, we can always express

$$
\left(\mathcal{T} g \mathcal{T}^{-1}-\mathbb{I}\right)-\left(\left(g^{*}\right)^{-1}-\mathbb{I}\right)=(u+t c)[B-t A],
$$

with $A=\left(\begin{array}{ll}0 & 1 \\ 0 & 0\end{array}\right)$ and $B=\left(\begin{array}{cc}-1 & 0 \\ 0 & 1\end{array}\right)$. Notice that $A, B$, satisfy the algebra

$$
[A, B]=2 A,
$$

which corresponds to a non-Abelian group $A(1)$ associated with the collinear transformations in one dimension (translation and scaling). The same algebra was already identified at the level of nine-dimensional type II gauged supergravity in the type IIA sector [13].

Notice that for the parabolic case $(a=1, b=\mathrm{p}, c=0$, $d=1$ ) both coefficients in Eq. (43) vanish, and the coinvariants and their duals lie in the same equivalence class.

Clearly the elliptic and hyperbolic coinvariant classes are mapped under $T_{U}$ duality into an inequivalent coinvariant equivalence class. $T_{U}$ duality acts nonlinearly on the supermembrane dual bundle, and this dual realization is associated with a non-Abelian algebra of the $A(1)$ group. We conjecture that the $A(1)$ M2-brane 2-torus bundle at low energies is described by the type IIA gauged supergravities corresponding to a non-Abelian $A(1)$.

\section{C. $U$-dual of M2-brane trombone torus bundles}

Trombone symmetry produces supergravities that do not have a Lagrangian but are uniquely defined through the equations of motion. The reason is that the trombone symmetry is not a symmetry of the action since it scales the Langrangian, but it is a symmetry of the equations of motion. At quantum level, however, there exists a welldefined action since it is possible to define an invariant hamiltonian. In type II supergravity in $9 \mathrm{D}$, the global symmetries are $G L(2, R)=S L(2, R) \times R$, the breaking of the group into its arithmetic subgroup $G L(2, Z)$ with determinant \pm 1 is not able to capture the effect of the scaling. Hence in order to obtain the scaling symmetries, an alternative procedure is needed. This question was in fact solved many years ago by Cremmer et al. [60] by means of a nonlinear realization of the group $S L(2, Z)$ that they called active $S L(2, Z)$. In Ref. [20] the authors used this realization to obtain a bundle description of the supermembrane with gauged trombone symmetry. In the following, in order to be self-contained, we first summarize those results, i.e., the realization of the trombone symmetry and their associated torus bundles, before performing the characterization of their duals.

Let us consider a nonlinear representation of the group $S L(2, Z)$ in terms of the $2 \times 2$ matrices $\mathbb{H}_{i j}$ as in Ref. [20]. Given two different charges $\mathbf{Q}_{i}, \mathbf{Q}_{j}$ labeled with two different indices $i, j$ and given $\mathbb{T}_{i j}$ as the $\operatorname{SL}(2, Z)$ active transformation, it acts on the charges as follows:

$$
\mathbb{H}_{i j} \mathbf{Q}_{i}=\mathbf{Q}_{j} \quad \text { and } \quad \frac{\mathbb{H}_{i j}}{h_{i j}}\left(\begin{array}{l}
\tau \\
1
\end{array}\right)=\left(\begin{array}{c}
\tau \\
1
\end{array}\right)
$$

The solution for Eq. (45) is given by

$$
\mathbb{U}_{j i}=\left(\begin{array}{cc}
-\frac{p_{j}}{q_{j}} U+\frac{q_{i}}{q_{j}} C & \frac{p_{i}}{q_{i}}+\frac{p_{i} p_{j}}{q_{i} q_{j}} U-\frac{p_{i}}{q_{j}} C \\
-U & \frac{q_{j}}{q_{i}}+\frac{p_{i}}{q_{i}} U
\end{array}\right),
$$

where

$h_{j i}=\frac{p_{j}-q_{j} \bar{\tau}}{p_{i}-q_{i} \bar{\tau}}, \quad U=\frac{p_{j} q_{i}-p_{i} q_{j}}{\left|p_{i}-q_{i} \bar{\tau}\right|^{2}}, \quad C=\frac{\left|p_{j}-q_{j} \tau\right|^{2}}{\left|p_{i}-q_{i} \tau\right|^{2}}$.

For each monodromy group there exists a unique nonlinear realization of it, $\mathbb{t}_{G}$. There are three nonlinear realizations associated with the elliptic, parabolic, and hyperbolic monodromy classes; however, they cannot be distinguish among them, so on the type IIB side we obtain only a single M2-brane trombone torus bundle class. This is in agreement with the fact that at low energies on the type IIB side there is a unique 9D trombone supergravity.

This transformation generates the complete lattice of charges for a given vacuum (that is, the asymptotic value of the scalar moduli). $\mathbb{H}_{j i} \in G L(2, R)$ is the nonlinear representation of $\mathcal{M}_{j i} \mathbf{Q}_{i}=\mathbf{Q}_{j}$, with $\mathcal{M}_{j i} \in S L(2, Z)$, which acts linearly on the charges, but nonlinearly on the moduli. The Hamiltonian $H$ is invariant since

$$
\tau \rightarrow \tau, \quad \mathbf{W} \rightarrow \mathbf{W}, \quad \mathrm{R} \rightarrow \mathrm{R} .
$$

While the mass operator changes since the KK contribution changes, $K K \rightarrow K K^{\prime}$ according to Eq. (45). See Ref. [20] for details. The structure of the supermembrane trombone bundle differs from those in which the monodromy is linearly realized since in the former the moduli is unaltered by the trombone monodromy. Consequently on the type IIB side each bundle contains only a single pair of winding charges $\mathbf{W}=\left(\begin{array}{l}l \\ m\end{array}\right)$ instead of an orbit, and therefore there is no monodromy associated with the base manifold. The only monodromy of the bundle is associated with the fiber $\mathcal{M}_{G}$, and it is nonlinearly realized in terms of $\mathbb{U}_{G}$. The coinvariant class of charges of the fiber is defined as

$$
C_{F}^{\text {tromb }}=\left\{\mathbf{Q}+\left(\mathbb{\boxplus}_{G}-\mathbb{I}\right) \hat{\mathbf{Q}}\right\},
$$

and the structure of the bundle in terms of the coinvariant classes is $\left(C_{F}^{\text {tromb }}, \mathbf{W}\right)$. 
The $T_{U}$-duality transformation for the trombone torus bundle maps the coinvariant class of the fiber of the original bundle into the coinvariant class of the base, and the original winding single charge of the base into a single dual KK charge of the dual fiber. As a result, the dual trombone bundle $\left(\tilde{C}_{B}^{\text {tromb }}, \mathbf{Q}\right)$ corresponds to a bundle that has a trivial monodromy on the fiber but a nontrivial monodromy in the base manifold with coinvariant classes given by

$$
\tilde{C}_{B}^{\text {tromb }}=C_{F}^{\text {tromb }},
$$

where $\tilde{\tau}$ and $\tilde{\mathrm{R}}$ the $T_{U}$-dual moduli parameters transform as in Eqs. (22) and (25), and they are defined by the covariant class. They do not depend on any particular element of the orbit. The geometric structure can be interpreted as a compatible set of fiber bundles with characteristic classes defined by the coinvariant class of winding matrices. Under the $T_{U}$-duality transformation the Hamiltonian which is invariant on the orbits of $\mathbb{U}_{G}$ is transformed to a Hamiltonian invariant under the orbits of $\mathbb{q}_{G}^{*}$ and the bundle change as $\left(C_{F}^{\text {tromb }}, \mathbf{W}\right) \stackrel{\mathbb{T}}{\rightarrow}\left(\tilde{C}_{B}^{\text {tromb }}, \tilde{\mathbf{Q}}\right)$.

We notice that in the expression of the Hamiltonian of the trombone torus bundle with coinvariant $C_{F}^{\text {trombone }}$, there is not a $\Theta$ matrix on the expression of the covariant derivative since $\mathbf{W}, \tau, \mathrm{R}$ remain fixed under the action of the nonlinear transformation $\mathbb{M}_{G}$. At low energies they correspond to gauged theories in nine dimensions obtained from the massive deformation of type IIA supergravity in 10D. However, there exists a $\Theta$ matrix in the covariant derivatives which compensates for the transformation of the winding matrix on the dual torus bundle (the type IIB side).

\section{Two inequivalent duals of the M2-brane trombone torus bundle}

The gauging is obtained by means of the nonlinear representation $\mathbb{\boxplus}^{G}$ with $\mathcal{M}_{G} \subset \mathcal{M}_{j i}$ associated with a particular monodromy equivalence class. To compute it, we particularize the monodromy matrix $\mathcal{M}_{G} \mathbf{Q}_{i}=\mathbf{Q}_{j}$, and we substitute in $\mathbb{U}_{j i}\left(\tau, q_{i}, p_{i}, q_{j}, p_{j}\right)$ and $\mathbf{Q}_{i}=\left(\begin{array}{l}p_{i} \\ q_{i}\end{array}\right)$ and $\mathbf{Q}_{j}$, respectively. The linear representation $\mathcal{M}_{j i}$ contains the three inequivalent conjugation classes (elliptic, parabolic, and hyperbolic); however, the nonlinear representation $\mathbb{U}_{j i}$ does not have any particular property which may distinguish the elliptic to the hyperbolic cases. Under $T_{U}$ duality in distinction with the previous case, there are two inequivalent types of dual principal bundles, one with the parabolic monodromy nonlinearly realized in the base, and a second one associated with the nonlinear realization elliptic and hyperbolic monodromy dual in the base manifold. In the case of parabolic monodromy, the duality matrix $\mathcal{T}$ commute with $\mathbb{H}_{i j}$. In the other two cases the $T_{U}$ duality does not commute with $\mathbb{H}_{i j}$, but since they cannot be distinguished, they form a second inequivalent class of trombone torus bundles on the type IIA side. Nicely at low
M2-brane "Type IIB origin"

M2-brane "Type IIA origin"

M2 T-duality

Parabolic

Parabolic

Trombone

Trombone I

Trombone II

\section{Elliptic}

Hyperbolic

Scaling $A(1)$

FIG. 1. The precise relations between the M2-brane bundle with monodromy in $S L(2, Z)$ inequivalent classes when a M2brane $T_{U}$ duality is performed.

energies they are in correspondence with the two trombone gauged supergravities of the type IIA sector. The precise relation among the inequivalent classes of M2-brane torus bundles with monodromy in $S L(2, Z)$ is illustrated on Fig. 1.

\section{DISCUSSION AND CONCLUSIONS}

We find eight independent inequivalent classes of supermembrane torus bundles with monodromy linearly and nonlinearly realized in $S L(2, Z)$. Four of them had previously been found, but those associated with the type IIA side are new. The M2-brane torus bundle is characterized by the monodromy, the coinvariants of the base manifold $C_{B}$, and that of the fiber $C_{F}$. Their Hamiltonians and mass operators for the nine different cases (eight inequivalent class of bundles with monodromy and one principal) are all invariant under the duality action that we have defined in this work irrespective on the monodromy group. However, the structure of the bundle, for arbitrary M2-brane torus bundles with monodromy, is not necessarily preserved. Only for the cases in which $U$ duality acts linearly (for example, for monodromy that is parabolic or trivial) is the bundle structure preserved. We observe that the monodromy group of the M2-brane torus bundle coincides with the gauging groups of type II supergravity. Hence we relate each of these inequivalent classes of M2-brane 2-torus bundles - with and without monodromy - to each of the eight type II gauged supergravities in 9D and the maximal one, respectively. Recently it was shown that the supermembrane theory with central charge condition (described by 2-torus bundles with monodromy) is equivalent to a supermembrane on a constant 3-form background toroidally compactified in the presence of fluxes [61]. Since flux compactifications are related to gauged supergravities, this is another way to evidence the relation between the 
M2-brane torus bundle with monodromy and its description at low energies in terms of gauged supergravities. We believe that the nonpreservation of the coinvariant equivalence classes under $U$ duality is the underlying reason for the differing structures between the type IIB and type IIA gauged supergravities in nine dimensions from the M-theory viewpoint. We have proved that the $U$ dual of the parabolic coinvariants remains in the same class of coinvariants; moreover, they seem to be the only ones that preserve the coinvariant class. Consequently the type IIB parabolic M2-brane bundle is mapped through duality into the parabolic type IIA M2-brane bundle. At low energies it becomes natural to relate it to the type IIA parabolic supergravity, which corresponds to the KK reduction of Romans supergravity in 10D to 9D.

The $U$-duals of the supermembrane with monodromies elliptic and hyperbolic do not preserve the coinvariant class, even though the dual monodromy is conjugated to the original one. $U$ duality acts nonlinearly on these M2-brane bundles, and it is responsible for changing the bundle class. The algebra of the monodromy groups elliptic and hyperbolic with the $U$-duality transformation form a non-Abelian algebra $A(1)$ that acts like a scaling and a translation. The dual M2-brane bundle with these two inequivalent classes of monodromies corresponds to a single class of dual M2-brane bundles that we call $A(1)$. At low energies we consider that it could be related it to the type IIA $A(1)$ gauged supergravity in 9D.

Trombone symmetry of the supermembrane torus bundle is a nonlinear realization of the $S L(2, Z)$ group. It acts linearly on the charges but nonlinearly on the moduli, and the bundle description once it is gauged is completely different from the previous cases considered. The M2-brane bundle with gauged trombone monodromy are classified according to the monodromy, but they contain only one class of coinvariants either of the fiber (type IIB side) or of the base (type IIA side). Under $U$ duality the nonlinear realization of the three inequivalent classes of M2-brane torus bundles (elliptic, hyperbolic, or parabolic subgroups), they form a unique M2-brane trombone bundle on the type IIB side. Under $U$ duality it maps onto two inequivalent classes of dual M2-brane trombone bundles on the type IIA side: one associated with the gauging of the nonlinear realization of the parabolic monodromy, and a second one associated with the gauging of the nonlinear realization of the bundles with elliptic and hyperbolic monodromy. Nicely these two inequivalent classes of $T$-bundles can naturally be associated on the 10D type IIA side with the KK reduction of the massive Howe, Lambert, and West supergravity, and to the SS reduction of type IIA maximal supergravity.

\section{ACKNOWLEDGMENTS}

J. M. P. is grateful to the Scientific Programme Stringy Geometry, Mainz Institute for Theoretical Physics (MITP) for its hospitality and its partial support during part of the realization of this work. M.P.G.d.M. is grateful to I. Cavero-Pelaez for the helpful comments on the manuscript. M. P. G. d. M. has been supported by Convenio Marco UES ANT1398 PROJECT and ANT1555 PROJECT, Plan plurianual de fortalecimiento Institucional, MECESUP, Chile. A. R. and M. P. G. d. M. are partially supported by Project No. FONDECYT 1161192 (Chile). M. P. G. d. M. and A. R. participated as external nodes of the EU-COST Action No. MP1210 "The String Theory Universe" during the realization of this work.

\section{APPENDIX A: COINVARIANTS}

Let us define the map associated with the coinvariant classes on the KK sector to the coinvariant classes on the winding sector. We take one element $\mathbf{Q}$ in a coinvariant class of the $\mathrm{KK}$ sector and map it to an element $\mathbf{W}$ of a coinvariant class of the winding sector. There exists an element of $S L(2, Z)$ with equal diagonal terms $\mathcal{T}$ which maps $\mathbf{Q}$ to $\mathbf{W}$ :

$$
\mathbf{W}=\mathcal{T} \mathbf{Q}
$$

In the case that we are considering here, where the Abelian group $G$ has only one generator $J$,

$$
g \in \mathcal{M}_{G}, \quad g=J^{a},
$$

where $a$ is an integer, then $g-\mathbb{I}=J^{a}-\mathbb{I}$. We can distinguish among three different cases:

if $a=0, \quad g-\mathbb{I}=0$,

if $a>0, \quad J^{a}-\mathbb{I}=(J-\mathbb{I})\left(J^{a-1}+J^{a-2}+\cdots+\mathbb{I}\right)$,

if $a<0, \quad J^{a}-\mathbb{I}=\left(\mathbb{I}-J^{-a}\right) J^{a}$.

Hence if $a=0$, we have one element $\mathbf{Q}$. If $a>0$, we have

$$
C_{F}=\{\mathbf{Q}+(J-1) \hat{\hat{\mathbf{Q}}}\},
$$

where $\hat{\hat{\mathbf{Q}}}=\left[J^{a-1}+J^{a-2}+\cdots+\mathbb{I}\right] \hat{\mathbf{Q}}$.

If $a=1$, we get $\hat{\mathbf{Q}}=\hat{\mathbf{Q}}$, and if $a \geq 2, \hat{\mathbf{Q}}$ belongs to a subset of the whole $\hat{\mathbf{Q}}$ sector. We then conclude that the coinvariant class can be expressed as

$$
C_{F}=\{\mathbf{Q}+(J-1) \hat{\mathbf{Q}}\} .
$$

The same result holds for $a<0$. Consequently without lose of generality we can take the elements of the coinvariant class as in Eq. (A5). Notice that the case $a=0$ is also contained in the class since it corresponds to considering $\hat{\mathbf{Q}}=\left(\begin{array}{l}0 \\ 0\end{array}\right)$. 


\section{APPENDIX B: GENERAL $T_{U}$-DUALITY TRANSFORMATION}

In this appendix we obtain the most general transformation of $T_{U}$ duality for the supermembrane $T$-bundles. Given a particular supermembrane wrapping the 2-torus target space, it has an associated matrix of windings $\mathbb{W}$ with determinant $\operatorname{det}(\mathbb{W})=\mathrm{n} \neq 0$ such that, applying an $\mathbb{S} \in S L(2, Z)$, it admits a triangular description,

$$
\mathbb{W} S=\left(\begin{array}{ll}
\mathrm{n} & e \\
0 & 1
\end{array}\right)
$$

Multiplying on the left-hand side by a parabolic matrix $\mathbb{R}$, the winding can be expressed in terms of its canonical form

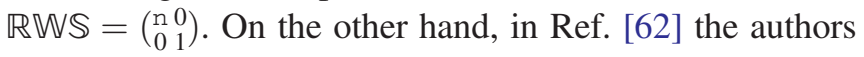
showed that there always exists a map $T_{U}$ duality that maps

$$
\mathbb{W S}=\mathcal{T} \mathbb{Q},
$$

where $\mathbb{Q}$ is the matrix of charge of determinant $n$ whose first row is $\mathbb{Q}$. $\mathcal{T}$, for sake of brevity, denotes the parabolic $T_{U}$-duality transformation $\mathcal{T}_{p}$. Then for arbitrary $\mathbb{W}, \mathbb{Q}$ charges we want to obtain the more general $\hat{S}, \mathcal{T}$ such that they satisfy $\mathbb{W} \hat{S}=\mathcal{T} \mathbb{Q}$. Consequently

$$
\mathbb{W}=\mathcal{T} \mathcal{T}^{-1} \mathbb{W} \mathbb{S} \hat{S}^{-1}
$$

We want to find the more general $u=\mathcal{T} \mathcal{T}^{-1}$ and $v=\mathbb{S S}^{-1}$ such that the above relation is satisfied. This condition is verified for

$$
\begin{aligned}
\mathcal{T} & =A B A^{-1}(\mathcal{T}) \\
& =\left(\begin{array}{cc}
d-e c & d t-b \mathrm{n}+e(a-d-c t-c e) \\
-c & -c(t-e)+a
\end{array}\right),
\end{aligned}
$$

with $A=\left(\begin{array}{ll}1 & e \\ 0 & 1\end{array}\right)$ and $B=\left(\begin{array}{cc}d & -b \mathrm{n} \\ -c & a\end{array}\right)$, with $A, B \in S L(2, Z)$.

One can distinguish among three different cases, according to the value of the trace

$$
\operatorname{Trace}(\mathcal{T})=d-c t+a,
$$

as elliptic, parabolic, and hyperbolic. For the case in which the central charge is restricted to be $n=1$, all of the three subclasses of $T_{U}$-duality matrices are allowed; however, this is not the more general case since for the wrapped supermembrane on a torus an arbitrary $\mathrm{n}$ is allowed.

(a) The parabolic case $\mathcal{T}_{p}=\left(\begin{array}{ll}1 & t \\ 0 & 1\end{array}\right)$ has already being discussed [57], it corresponds to have $a=1, d=1$ with $c=0$ and it is satisfied for any configuration of charges, windings and central charge, so in the following we will analyze the two other possibilities.

(b) The elliptic case corresponds to have $c= \pm 1$ and $b \mathrm{n}= \pm 1-e(e-t)$ since it must verify that the two trace entries are equal. For arbitrary central charge $\mathrm{n} \neq 1$ the above equation is not satisfied apart for solutions associated with particular values of charges and windings.

(c) For the hyperbolic case we will analyze separately three different cases restricted to assume that $n$ is prime:

(i) In first place consider $(2 e-t)$ and $\mathrm{n}$ relatively prime integers other than zero. Then choose $a$, $b$ such that the following relation is satisfied,

$$
(2 e-t)-b, \quad \text { then } \mathrm{n}=1, \quad c=1-a^{2} .
$$

In this case the trace is equal to $2\left(1-a^{2}\right)(e-t)+a$. Then if $a, b$ are the solutions, then $a+\lambda \mathrm{n}, b+\lambda(2 e-t)$ is also a solution. We always can find a solution of reversed sign to $(e-t)$. Then $\left(1-a^{2}\right)(e-t)$ and $a$ have the same sign, and consequently we need to choose a $\lambda$ large enough to guarantee only that

$$
|a+\lambda \mathrm{n}|>1
$$

and it corresponds to an hyperbolic solution. A hyperbolic solution for this first case always exists.

(ii) If $(2 e-t)$ and $\mathrm{n}$ other than zero are not relatively prime, then $(2 e-t)=m \mathrm{n}$ with $m \neq 0$. Substituting in the relation $a d-b c \mathrm{n}=1$, one obtains $a^{2}+c(a m-b) \mathrm{n}=1$. There is a solution $b=$ am with $a=1$ and an arbitrary $c$. The trace is

$$
\operatorname{Tr}(\mathcal{T})=2(e-t) c+a \geq 2 .
$$

One can choose $c=e-t$, and then the trace corresponds to a hyperbolic matrix. The case $e=t$ (which would not correspond to a hyperbolic case) cannot happen since, in the case we consider, $2 e-t=m \mathrm{n}$. Then $e=m \mathrm{n}$ but $e<\mathrm{n}$ always, as shown in Ref. [46]. Consequently a hyperbolic matrix for this case always exists.

(iii) The last case to consider occurs when $2 e-k=0$. Then $a=\mathrm{n}+1, b=\varepsilon=\operatorname{sign}(e-k), c=\varepsilon(\mathrm{n}+2)$ with trace

$\operatorname{Tr}(\mathcal{T})=2[\varepsilon(\mathrm{n}+29(e-k))+a]>2(\mathrm{n}+1)>2$.

Consequently a hyperbolic matrix for this case always exists. 
In conclusion there always exists a parabolic transformation $\mathcal{T}$ and a hyperbolic transformation $\mathcal{T}_{h}$ inside the set of $T_{U}$-duality transformations allowed for any value of the central charge $\mathrm{n}$. Each hyperbolic transformation has a different trace for every different set of charges.
[1] L. Boulton, M. P. Garcia del Moral, and A. Restuccia, The ground state of the $D=11$ supermembrane and matrix models on compact regions, Nucl. Phys. B910, 665 (2016).

[2] E. Cremmer, B. Julia, and J. Scherk, Supergravity theory in eleven-dimensions, Phys. Lett. 76B, 409 (1978).

[3] E. Cremmer and B. Julia, The $\mathrm{SO}(8)$ supergravity, Nucl. Phys. B159, 141 (1979).

[4] E. Bergshoeff, E. Sezgin, and P. K. Townsend, Supermembranes and eleven-dimensional supergravity, Phys. Lett. B 189, 75 (1987).

[5] B. de Wit, H. Nicolai, and H. Samtleben, Gauged supergravities, tensor hierarchies, and M-theory, J. High Energy Phys. 02 (2008) 044.

[6] S. J. Gates, H. Nishino, and E. Sezgin, Supergravity in $D=9$ and its coupling to non-compact sigma-models, Classical Quantum Gravity 3, 21 (1986).

[7] H. Samtleben, Lectures on gauged supergravity and flux compactifications, Classical Quantum Gravity 25, 214002 (2008).

[8] M. Trigiante, Gauged supergravities, Phys. Rep. 680, 1 (2017).

[9] J. J. Fernandez-Melgarejo, T. Ortin, and E. Torrente-Lujan, The general gaugings of maximal $d=9$ supergravity, J. High Energy Phys. 10 (2011) 068.

[10] E. Bergshoeff, M. de Roo, and E. Eyras, Gauged supergravity from dimensional reduction, Phys. Lett. B 413, 70 (1997).

[11] P. Meessen and T. Ortin, An SL(2,Z) multiplet of ninedimensional type II supergravity theories, Nucl. Phys. B541, 195 (1999).

[12] J. Gheerardyn and P. Meessen, Supersymmetry of massive $D=9$ supergravity, Phys. Lett. B 525, 322 (2002).

[13] E. Bergshoeff, T. de Wit, U. Gran, R. Linares, and D. Roest, (Non-)abelian gauged supergravities in nine dimensions, J. High Energy Phys. 10 (2002) 061.

[14] S. J. Gates and B. Zwiebach, Gauged and ungauged $N=4$ supergravities in superspace, Report No. CALT-68-943, 1982.

[15] S. J. Gates and B. Zwiebach, Gauged $N=4$ supergravity theory with a new scalar potential, Phys. Lett. 123B, 200 (1983).

[16] B. Biran, F. Englert, B. de Wit, and H. Nicolai, Gauged $N=8$ supergravity and its breaking from spontaneous compactification, Phys. Lett. 124B, 45 (1983).

[17] C. M. Hull, Massive string theories from M-theory and F-theory, J. High Energy Phys. 11 (1998) 027.

[18] I. V. Lavrinenko, H. Lu, and C. N. Pope, From topology to generalized dimensional reduction, Nucl. Phys. B492, 278 (1997).
[19] I. V. Lavrinenko, H. Lu, and C. N. Pope, Fiber bundles and generalized dimensional reduction, Classical Quantum Gravity 15, 2239 (1998).

[20] M. P. Garcia del Moral, J. M. Pena, and A. Restuccia, Supermembrane origin of type II gauged supergravities in 9D, J. High Energy Phys. 09 (2012) 063.

[21] L. J. Romans, Massive $N=2$ a supergravity in ten dimensions, Phys. Lett. 169B, 374 (1986).

[22] P. S. Howe, N. D. Lambert, and P. C. West, A new massive type IIA supergravity from compactification, Phys. Lett. B 416, 303 (1998).

[23] C. Hull and B. Zwiebach, Double field theory, J. High Energy Phys. 09 (2009) 099.

[24] A. Tseytlin, Duality symmetric formulation of string world sheet dynamics, Phys. Lett. B 242, 163 (1990).

[25] M. Duff, Duality rotations in string theory, Nucl. Phys. B335, 610 (1990).

[26] D. S. Berman, E. T. Musaev, and D. C. Thompson, Duality invariant M-theory: Gauged supergravities and ScherkSchwarz reductions, J. High Energy Phys. 10 (2012) 174.

[27] M. J. Duff and J. X. Lu, Duality rotations in membrane theory, Nucl. Phys. B347, 394 (1990).

[28] D. C. Thompson, Duality invariance: From M-theory to double field theory, J. High Energy Phys. 08 (2011) 125.

[29] N. Hitchin, Generalized Calabi-Yau manifolds, Q. J. Mech. Appl. Math. 54, 281 (2003).

[30] J. Shelton, W. Taylor, and B. Wecht, Nongeometric flux compactifications, J. High Energy Phys. 10 (2005) 085.

[31] R. A. Reid-Edwards, Flux compactifications, twisted tori and doubled geometry, J. High Energy Phys. 06 (2009) 085.

[32] C. M. Hull, Global aspects of $T$-duality, gauged sigma models and T-folds, J. High Energy Phys. 10 (2007) 057.

[33] G. Aldazabal, E. Andres, P. G. Camara, and M. Grana, $U$-dual fluxes and generalized geometry, J. High Energy Phys. 11 (2010) 083.

[34] D. S. Berman and M. J. Perry, Generalized geometry and M theory, J. High Energy Phys. 06 (2011) 074.

[35] C. D. Blair, E. Malek, and J.-H. Park, M-theory and type IIB from a duality manifest action, J. High Energy Phys. 01 (2014) 172.

[36] D. Marques and C. A. Nunez, $T$-duality and $\alpha^{\prime}$-corrections, J. High Energy Phys. 10 (2015) 084.

[37] G. Aldazabal, M. Grana, S. Iguri, M. Mayo, C. Nuñez, and J. A. Rosabal, Enhanced gauge symmetry and winding modes in double field theory, J. High Energy Phys. 03 (2016) 093.

[38] G. Aldazabal, D. Marques, and C. Nunez, Double field theory: A pedagogical review, Classical Quantum Gravity 30, 163001 (2013). 
[39] G. Aldazabal, W. Baron, D. Marques, and C. Nunez, The effective action of double field theory, J. High Energy Phys. 11 (2011) 052.

[40] G. Aldazabal, W. Baron, D. Marques, and C. Nunez, Erratum: the effective action of double field theory, J. High Energy Phys. 11 (2011) 109(E).

[41] D. Geissbuhler, D. Marques, C. Nunez, and V. Penas, Exploring double field theory, J. High Energy Phys. 06 (2013) 101.

[42] E. Alvarez, L. Alvarez-Gaume, J. L. F. Barbon, and Y. Lozano, Some global aspects of duality in string theory, Nucl. Phys. B415, 71 (1994).

[43] H. Lu, C. N. Pope, and K. S. Stelle, Multiplet structures of BPS solitons, Classical Quantum Gravity 15, 537 (1998).

[44] V. Bengtsson, M. Cederwall, H. Larsson, and B. E. W. Nilsson, $U$-duality covariant membranes, J. High Energy Phys. 02 (2005) 020.

[45] J. H. Schwarz, An SL(2,Z) multiplet of type IIB superstrings, Phys. Lett. B 360, 13 (1995); Erratum, 364, 252(E) (1995).

[46] M. P. Garcia del Moral, I. Martin, and A. Restuccia, Nonperturbative $\mathrm{SL}(2, \mathrm{Z})(\mathrm{p}, \mathrm{q})$-strings manifestly realized on the quantum M2, arXiv:0802.0573.

[47] I. Martin, J. Ovalle, and A. Restuccia, D-branes, symplectomorphisms and noncommutative gauge theories, Nucl. Phys. B, Proc. Suppl. 102, 169 (2001); Compactified $D=11$ supermembranes and symplectic noncommutative gauge theories, Phys. Rev. D 64, 046001 (2001).

[48] B. de Wit, J. Hoppe, and H. Nicolai, On the quantum mechanics of supermembranes, Nucl. Phys. B305, 545 (1988).

[49] I. Martin and A. Restuccia, Magnetic monopoles over topologically nontrivial Riemann surfaces, Lett. Math. Phys. 39, 379 (1997).

[50] M. P. Garcia del Moral and A. Restuccia, On the spectrum of a noncommutative formulation of the $D=11$ supermembrane with winding, Phys. Rev. D 66, 045023 (2002).
[51] I. Martin, A. Restuccia, and R. S. Torrealba, On the stability of compactified $D=11$ supermembranes, Nucl. Phys. B521, 117 (1998).

[52] L. Boulton, M. P. Garcia del Moral, and A. Restuccia, Discreteness of the spectrum of the compactified $D=11$ supermembrane with non-trivial winding, Nucl. Phys. B671, 343 (2003).

[53] L. Boulton, M. P. Garcia del Moral, and A. Restuccia, Spectral properties in supersymmetric matrix models, Nucl. Phys. B856, 716 (2012).

[54] M. P. Garcia del Moral, I. Martin, J. M. Pena, and A. Restuccia, SL(2,Z) symmetries, supermembranes and symplectic torus bundles, J. High Energy Phys. 09 (2011) 068.

[55] M. P. Garcia del Moral, On the geometry of sculptinglike gauging processes, Int. J. Geom. Methods Mod. Phys. 11, 1460040 (2014).

[56] P. J. Khan, Symplectic torus bundles and group extensions, N. Y. J. Math. 11, 35 (2005); arXiv:math/0405109.

[57] M. P. Garcia del Moral, J. M. Pena, and A. Restuccia, $T$-duality invariance of the supermembrane, Int. J. Geom. Methods Mod. Phys. 10, 1360010 (2013).

[58] C. M. Hull, Gauged $D=9$ supergravities and ScherkSchwarz reduction, Classical Quantum Gravity 21, 509 (2004).

[59] A. Dabholkar and C. Hull, Duality twists, orbifolds, and fluxes, J. High Energy Phys. 09 (2003) 054.

[60] E. Cremmer, H. Lü, C. N. Pope, and K. S. Stelle, Spectrumgenerating symmetries for BPS solitons, Nucl. Phys. B520, 132 (1998).

[61] M. P. Garcia del Moral, C. Las Heras, P. Leon, J. M. Pena, and A. Restuccia, Supermembrane theory on a curved constant background, arXiv:1811.11231.

[62] M. P. Garcia del Moral, J. M. Pena, and A. Restuccia, Aspects of the $T$-duality construction for the supermembrane theory, J. Phys. Conf. Ser. 720, 012025 (2016). 